\title{
Universally Composable Multi-party Computation with an Unreliable Common Reference String*
}

\author{
Vipul Goyal ${ }^{1, \star \star}$ and Jonathan Katz ${ }^{2, \star \star \star}$ \\ ${ }^{1}$ Department of Computer Science, UCLA \\ vipul@cs.ucla.edu \\ ${ }^{2}$ Department of Computer Science, University of Maryland \\ jkatz@cs. umd.edu
}

\begin{abstract}
Universally composable (UC) multi-party computation has been studied in two settings. When a majority of parties are honest, UC multi-party computation is possible without any assumptions. Without a majority of honest parties, UC multi-party computation is impossible in the plain model, but feasibility results have been obtained in various augmented models. The most popular such model posits a common reference string (CRS) available to parties executing the protocol.

In either of the above settings, some assumption regarding the protocol execution is made: i.e., that many parties are honest in the first case, or that a legitimately-chosen string is available in the second. If this assumption is incorrect then all security is lost.

A natural question is whether it is possible to design protocols secure if either one of these assumptions holds, i.e., a protocol which is secure if either at most $s$ players are dishonest or if up to $t>s$ players are dishonest but the CRS is chosen in the prescribed manner. We show that such protocols exist if and only if $s+t<n$.
\end{abstract}

\section{Introduction}

Protocols proven to satisfy the definition of universal composability 5] offer strong and desirable security guarantees. Informally speaking, such protocols remain secure even when executed concurrently with arbitrary other protocols running in some larger network, and can be used as sub-routines of larger protocols in a modular fashion.

Universally composable (UC) multi-party computation of arbitrary functionalities has been investigated in two settings. When a majority of the parties running a protocol are assumed to be honest, UC computation of arbitrary functionalities is possible without any cryptographic assumptions. (This is claimed

\footnotetext{
^ This work was done in part while the authors were visiting IPAM.

** Research supported in part by NSF ITR and Cybertrust programs (including grants \#0430254, \#0627781, \#0456717, and \#0205594).

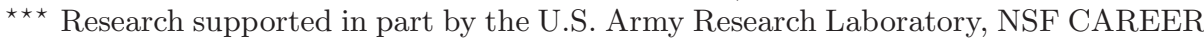
award \#0447075, and US-Israel Binational Science Foundation grant \#2004240.
} 
in [5], building on 317].) This result holds in the so-called "plain model" which assumes only pairwise private and authenticated channels between each pair of parties. (A broadcast channel or a PKI are not needed [10, since fairness and output delivery are not guaranteed in the UC framework.)

In contrast, when the honest players cannot be assumed to be in the majority, it is known that UC computation of general functions is not possible in the plain model regardless of any cryptographic assumptions made. Canetti and Fischlin [7] showed the impossibility of two-party protocols for commitment and zero knowledge, and Canetti, Kushilevitz, and Lindell 8 ruled out UC two-party computation of a wide class of functionalities.

To circumvent these far-reaching impossibility results, researchers have investigated various augmented models in which $\mathrm{UC}$ computation without honest majority might be realizable [5/7,9,1,12,6 15. The most widely-used of these augmented models is the one originally suggested by Canetti and Fischlin [7, in which a common reference string (CRS) is assumed to be available to all parties running a given execution of a protocol. (The use of a common reference string in cryptographic protocols has a long history that can be traced back to [4.) Canetti and Fischlin show that UC commitments and zero knowledge are possible in the two-party setting when a CRS is available, and later work of Canetti et al. 9] shows that (under suitable cryptographic assumptions) a CRS suffices for UC multi-party computation of arbitrary functionalities.

In summary, there are two types of what we might term "assumptions about the world" under which UC multi-party computation is known to be possible:

- When a strict minority of players are dishonest.

- When an arbitrary number of players may be dishonest, but a trusted CRS (or some other setup assumption) is available.

Our contribution. Known protocols designed under one of the assumptions listed above are completely insecure in case the assumption turns out to be false. For example, the BGW protocol [3] — which is secure when a majority of the parties are honest — is completely insecure in case half or more of the parties are dishonest. Similarly, the CLOS protocol 9] — which is secure for an arbitrary number of corrupted parties when a trusted CRS is available is completely insecure in the presence of even a single corrupted party if the protocol is run using a CRS $\sigma$ that is taken from the wrong distribution or, even worse, adversarially generated. Given this state of affairs, a natural question is whether it is possible to design a single protocol $\Pi$ that uses a common reference string $\sigma$ and simultaneously guarantees the following:

- Regardless of how $\sigma$ is generated (and, in particular, even if $\sigma$ is generated adversarially), $\Pi$ is secure as long as at most $s$ parties are corrupted.

- If $\sigma$ is generated "honestly" (i.e., by a trusted third party according to the specification), then $\Pi$ is secure as long as at most $t$ parties are corrupted.

In this case, we will call the protocol $\Pi$ an " $(s, t)$-secure protocol". It follows from [78] that $(s, t)$-security for general functionalities is only potentially achievable if 
$s<n / 2$, where $n$ is the total number of parties running the protocol. A priori, we might hope to achieve the "best possible" result that $(\lfloor(n-1) / 2\rfloor, n-1)$-secure protocols exist for arbitrary functionalities.

Here, we show tight positive and negative answers to the above question. First, we show that for any $s+t<n$ (and $s<n / 2$ ) there exists an $(s, t)$-secure protocol realizing any functionality. We complement this by showing that this is, unfortunately, the best possible: if $s+t=n$ then there is a large class of functionalities (inherited, in some sense, from [8]) for which no $(s, t)$-secure protocol exists. We prove security under adaptive corruptions for our positive result, while our negative result holds even for the case of non-adaptive corruptions.

For $n$ odd, the extremes of our positive result (i.e., $s=t=\lfloor(n-1) / 2\rfloor$, or $s=0, t=n-1)$ correspond to, respectively, a protocol secure for honest majority (but relying on cryptographic assumptions) or one secure against an arbitrary number of malicious parties but requiring a CRS. (For $n$ even we obtain a protocol that tolerates $s=\lfloor(n-1) / 2\rfloor$ corruptions regardless of how the CRS is constructed, and $t=s+1$ corruptions if the CRS is honestly-generated.) Our results also exhibit new protocols in between these extremes. Choice of which protocol to use reflects a tradeoff between the level of confidence in the CRS and the number of corruptions that can be tolerated: e.g., choosing $s=0$ represents full confidence in the CRS, while setting $s=t=\lfloor(n-1) / 2\rfloor$ means that there is effectively no confidence in the CRS at all.

Related work. Another suggestion for circumventing the impossibility results of 718 has been to use a definition of security where the ideal-model simulator is allowed to run in super-polynomial time [162]. This relaxation is sufficient to bypass the known impossibility results and leads to constructions of protocols for any functionality without setup assumptions. While these constructions seem to supply adequate security for certain applications, they require stronger (subexponential time) complexity assumptions and can be problematic when used as sub-routines within larger protocols.

Some other recent work has also considered the construction of protocols having "two tiers" of security. Barak, Canetti, Nielsen, and Pass [1] show a protocol relying on a key-registration authority: if the key-registration authority acts honestly the protocol is universally composable, while if this assumption is violated the protocol still remains secure in the stand-alone sense. Ishai et al. [13] and Katz [14, in the stand-alone setting, studied the question of whether there exist protocols that are "fully-secure" (i.e., guaranteeing privacy, correctness, and fairness) in the presence of a dishonest minority, yet still "secure-with-abort" otherwise. While the motivation in all these cases is similar, the problems are different and, in particular, a solution to our problem does not follow from (or rely on) any of these prior results.

Groth and Ostrovsky 11 recently introduced the multi-CRS model for universally composable multi-party computation. In this model, roughly speaking, the parties have access to a set of $k$ common reference strings, some $k^{\prime}$ of which are "good" (i.e., guaranteed to have been chosen honestly). The remaining $k-k^{\prime}$ strings are "bad", and can be chosen in an arbitrary manner. (Of course, it is 
not known which strings are "good" and which are "bad".) Groth and Ostrovsky explore conditions on $k, k^{\prime}$ under which UC multi-party computation is still possible. Although in both their case and our own the question boils down to what security guarantees can be achieved in the presence of a "bad" CRS, our end results are very different. In the work of Groth and Ostrovsky the number of corruptions to be tolerated is fixed and there are assumed to be some minimal number $k^{\prime}$ of "good" strings among the $k$ available ones. In our work, in contrast, it is possible that no "good" CRS is available at all; even in this case, though, we would still like to ensure security against some (necessarily) smaller set of corrupted parties. On the other hand, we do rely on the Groth-Ostrovsky result as a building block for our positive result.

\section{Preliminaries}

\subsection{Review of the UC Framework}

We give a brief overview of the UC framework, referring the reader to [5] for further details. The UC framework allows for defining the security properties of cryptographic tasks so that security is maintained under general composition with an unbounded number of instances of arbitrary protocols running concurrently. In the UC framework, the security requirements of a given task are captured by specifying an ideal functionality run by a "trusted party" that obtains the inputs of the participants and provides them with the desired outputs. Informally, then, a protocol securely carries out a given task if running the protocol in the presence of a real-world adversary amounts to "emulating" the desired ideal functionality.

The notion of emulation in the UC framework is considerably stronger than that considered in previous models. As usual, the real-world model includes the parties running the protocol and an adversary $\mathcal{A}$ who controls their communication and potentially corrupts parties, while the ideal-world includes a simulator $\mathcal{S}$ who interacts with an ideal functionality $\mathcal{F}$ and dummy players who simply send input to/receive output from $\mathcal{F}$. In the UC framework, there is also an additional entity called the environment $\mathcal{Z}$. This environment generates the inputs to all parties, observes all their outputs, and interacts with the adversary in an arbitrary way throughout the computation. A protocol $\Pi$ is said to securely realize an ideal functionality $\mathcal{F}$ if for any real-world adversary $\mathcal{A}$ that interacts with $\mathcal{Z}$ and real players running $\Pi$, there exists an ideal-world simulator $\mathcal{S}$ that interacts with $\mathcal{Z}$, the ideal functionality $\mathcal{F}$, and the "dummy" players communicating with $\mathcal{F}$, such that no poly-time environment $\mathcal{Z}$ can distinguish whether it is interacting with $\mathcal{A}$ (in the real world) or $\mathcal{S}$ (in the ideal world). $\mathcal{Z}$ thus serves as an "interactive distinguisher" between a real-world execution of the protocol $\Pi$ and an ideal execution of functionality $\mathcal{F}$. A key point is that $\mathcal{Z}$ cannot be re-wound by $\mathcal{S}$; in other words, $\mathcal{S}$ must provide a so-called "straight-line" simulation.

The following universal composition theorem is proven in [5]. Consider a protocol $\Pi$ that operates in the $\mathcal{F}$-hybrid model, where parties can communicate as 
usual and in addition have ideal access to an unbounded number of copies of the functionality $\mathcal{F}$. Let $\rho$ be a protocol that securely realizes $\mathcal{F}$ as sketched above, and let $\Pi^{\rho}$ be identical to $\Pi$ with the exception that the interaction with each copy of $\mathcal{F}$ is replaced with an interaction with a separate instance of $\rho$. Then $\Pi$ and $\Pi^{\rho}$ have essentially the same input/output behavior. In particular, if $\Pi$ securely realizes some functionality $\mathcal{G}$ in the $\mathcal{F}$-hybrid model then $\Pi^{\rho}$ securely realizes $\mathcal{G}$ in the standard model (i.e., without access to any functionality).

\subsection{Definitions Specific to Our Setting}

We would like to model a single protocol $\Pi$ that uses a CRS $\sigma$, where $\sigma$ either comes from a trusted functionality $\mathcal{F}_{C R S}$ (defined as in [7] and all subsequent work on UC computation in the CRS model) or is chosen in an arbitrary manner by the environment $\mathcal{Z}$. A technical detail is that parties running $\Pi$ can trivially "tell" where $\sigma$ comes from depending on which incoming communication tape $\sigma$ is written on (since an ideal functionality would write inputs to a different tape than $\mathcal{Z}$ would). Because this does not correspond to what we are attempting to model in the real world, we need to effectively "rule out" protocols that utilize this additional knowledge. The simplest way to do this is to define a "malicious CRS" functionality $\mathcal{F}_{m C R S}$ that we now informally describe. Functionality $\mathcal{F}_{m C R S}$ takes input $\sigma$ from the adversary $\mathcal{A}$ and then, when activated by any party $P_{i}$, sends $\sigma$ to that party. The overall effect of this is that $\mathcal{A}$ (and hence $\mathcal{Z})$ can set the CRS to any value of its choice; however, it is forced to provide the same value to all parties running protocol $\Pi$. When the parties interact with $\mathcal{F}_{C R S}$, this (intuitively) means that the CRS is "good"; when they interact with $\mathcal{F}_{m C R S}$ the CRS is "bad". We refer to this setting, where parties interact with either $\mathcal{F}_{C R S}$ or $\mathcal{F}_{m C R S}$ but do not know which, as the mixed CRS model. We can now define an $(s, t)$-secure protocol.

Definition 1. We say a protocol $\Pi(s, t)$-securely realizes a functionality $\mathcal{F}$ in the mixed CRS model if

(a) $\Pi$ securely realizes $\mathcal{F}$ in the $\mathcal{F}_{m C R S}$-hybrid model when at most s parties are corrupted.

(b) $\Pi$ securely realizes $\mathcal{F}$ in the $\mathcal{F}_{C R S}$-hybrid model when at most $t$ parties are corrupted.

We stress that $\Pi$ itself does not "know" in which of the two hybrid models it is being run. $\mathcal{S}$, however, may have this information hard-wired in. More concretely: although $\Pi$ is a fixed protocol, two different ideal-world adversaries $\mathcal{S}, \mathcal{S}^{\prime}$ may be used in proving each part of the definition above.

\section{Positive Result for $s+t<n$}

We begin by showing our positive result: if $s+t<n$ and $s<n / 2$ (where $n$ is the total number of parties running the protocol), then essentially any functionality 
$\mathcal{F}$ can be $(s, t)$-securely realized in the mixed CRS model. This is subject to two minor technical conditions 9] we discuss briefly now.

Non-trivial protocols. The ideal process does not require the ideal-process adversary to deliver the messages that are sent between the ideal functionality and the parties. A corollary of the above fact is that a protocol that "hangs" (i.e., never sends any messages and never generates output) securely realizes any ideal functionality. However, such a protocol is uninteresting. Following 9, we therefore let a non-trivial protocol be one for which all parties generate output if the real-life adversary delivers all messages and all parties are honest.

Well-formed functionalities. A well-formed functionality is oblivious of the corruptions of parties, runs in polynomial time, and reveals the internal randomness used by the functionality to the ideal-process adversary in case all parties are corrupted 9]. This class contains all functionalities we can hope to securely realize from a non-trivial protocol in the presence of adaptive corruptions, as discussed in [9].

We can now formally state the result of this section:

Theorem 1 Fix $s, t, n$ with $s+t<n$ and $s<n / 2$. Assume that enhanced trapdoor permutations, augmented non-committing encryption schemes, and dense cryptosystems exist. Then for every well-formed $n$-party functionality $\mathcal{F}$, there exists a non-trivial protocol $\Pi$ which $(s, t)$-securely realizes $\mathcal{F}$ against adaptive adversaries in the mixed CRS model.

The cryptographic assumptions of the theorem are inherited directly from [9], and we refer the reader there for formal definitions of each of these. Weaker assumptions suffice to achieve security against static corruptions; see [9].

To prove the above theorem, we rely on the results of Groth and Ostrovsky regarding the multi-CRS model [11. Informally, they show the following result: Assume parties $P_{1}, \ldots, P_{n}$ having access to $k \geq 1$ strings $\sigma_{1}, \ldots, \sigma_{k}$. As long as $k^{\prime}>k / 2$ of these strings are honestly generated according to some specified distribution $\mathcal{D}$ (and assuming the same cryptographic assumptions of the theorem stated above), then for every well-formed functionality $\mathcal{F}$ there exists a non-trivial protocol $\Pi$ securely realizing $\mathcal{F}$. We stress that the remaining $k-k^{\prime}$ strings can be generated arbitrarily (i.e., adversarially), even possibly depending on the $k^{\prime}$ honestly-generated strings.

Building on the above result, we now describe our construction. We assume there are $n$ parties $P_{1}, \ldots, P_{n}$ who wish to run a protocol to realize a (wellformed) functionality $\mathcal{F}$. Construct a protocol $\Pi$ as follows:

1. All parties begin with the same string $\sigma^{*}$ provided as input. (Recall the parties do not know whether this is a "good" CRS or a "bad" CRS.) $P_{1}, \ldots, P_{n}$ first "amplify" the given string $\sigma^{*}$ to $m$ CRSs $\sigma_{1}^{*}, \ldots, \sigma_{m}^{*}$, where $m$ is a parameter which is defined later on. The requirements here are simply that if $\sigma^{*}$ is "good", then each of $\sigma_{1}^{*}, \ldots, \sigma_{m}^{*}$ should be "good" also. (If $\sigma^{*}$ is "bad" then we impose no requirements on $\sigma_{1}^{*}, \ldots, \sigma_{m}^{*}$.) 
The above can be accomplished by using the CLOS protocol [9] as follows. Define an ideal functionality $\mathcal{F}_{m_{-} \text {new_CRS }}$ which generates $m$ new CRSs from the appropriate distribution $\mathcal{D}$ (where $\mathcal{D}$ refers to the the distribution used in the Groth-Ostrovsky result mentioned above) and outputs these to all parties. We use the CLOS protocol to realize the functionality $\mathcal{F}_{m_{-} n e w \_C R S}$. When running the CLOS protocol, use the given string $\sigma^{*}$ as the CRS.

Note that when $\sigma^{*}$ was produced by $\mathcal{F}_{C R S}$, security of the CLOS protocol guarantees that the $m$ resulting CRSs are all chosen appropriately. On the other hand, there are no guarantees in case $\sigma^{*}$ was produced by $\mathcal{F}_{m C R S}$, but recall that we do not require anything in that case anyway.

2. Following the above, each party $P_{i}$ chooses a string $\sigma_{i}$ according to distribution $\mathcal{D}$ (where, again, $\mathcal{D}$ is the distribution used in the Groth-Ostrovsky result mentioned above), and broadcasts $\sigma_{i}$ to all other parties 1

3. Each party receives $\sigma_{1}, \ldots, \sigma_{n}$, and sets $\sigma_{m+i}^{*}=\sigma_{i}$ for $i=1$ to $n$.

4. All parties now have $n+m$ strings $\sigma_{1}^{*}, \ldots, \sigma_{n+m}^{*}$. These strings are used to run the Groth-Ostrovsky protocol for $\mathcal{F}$.

We claim that for any $s, t$ satisfying the conditions of Theorem 1 , it is possible to set $m$ so as to obtain a protocol $\Pi$ that $(s, t)$-securely realizes $\mathcal{F}$. The conditions we need to satisfy are as follows:

- When $\Pi$ is run in the $F_{C R S}$-hybrid model, $\sigma^{*}$ is a "good" CRS and so the strings $\sigma_{1}^{*}, \ldots, \sigma_{m}^{*}$ are also "good". The $n-t$ honest parties contribute another $n-t$ "good" strings in step 2, above, for a total of $m+n-t$ "good" strings in the set of strings $\sigma_{1}^{*}, \ldots, \sigma_{n+m}^{*}$. At most $t$ of the strings in this set (namely, those contributed by the $t$ malicious parties) can be "bad". For the Groth-Ostrovsky result to apply, we need $m+n-t>t$ or

$$
m>2 t-n \text {. }
$$

- When $\Pi$ is run in the $F_{m C R S}$-hybrid model, $\sigma^{*}$ is adversarially-chosen and so we must assume that the strings $\sigma_{1}^{*}, \ldots, \sigma_{m}^{*}$ are also "bad". In step 2 , the malicious parties contribute another $s$ "bad" strings (for a total of $m+s$ "bad" strings), while the $n-s$ honest parties contribute $n-s$ "good" strings. For the Groth-Ostrovsky result to apply, we now need $n-s>m+s$ or

$$
m<n-2 s .
$$

Since $m, t, n$ are all integers, Equations (1) and (2) imply

$$
2 t-n \leq n-2 s-2
$$

or $s+t \leq n-1$. When this condition holds, the equations can be simultaneously satisfied by setting $m=n-2 s-1$, which gives a positive solution if $s<n / 2$.

The security of the above construction follows from the security of the GrothOstrovsky protocol [1] (the details are omitted).

${ }^{1}$ The "broadcast" used here is the UC broadcast protocol from 10 (which achieves a weaker definition than "standard" broadcast, but suffices for constructing protocols in the UC framework). 


\section{Impossibility Result for $s+t \geq n$}

In this section, we state and prove our main impossibility result which shows that the results of the previous section are tight.

Theorem 2 Let $n, t, s$ be such that $s+t \geq n$. Then there exists a well-formed deterministic functionality for which no non-trivial n-party protocol exists that $(s, t)$-securely realizes $\mathcal{F}$ in the mixed CRS model.

We in fact show that the above theorem holds for a large class of functionalities. That is, there exists a large class of functionalities for which no such non-trivial protocol exists.

The proof of Theorem 2 relies on ideas from the impossibility result of Canetti, Kushilevitz, and Lindell [8] that applies to 2-party protocols in the plain model. Since ours is inherently a multi-party scenario, our proof proceeds in two stages. In the first stage of our proof, we transform any $n$-party protocol $\Pi$ that securely computes a function $f$ in the mixed CRS model, into a two-party protocol $\Sigma$ in the mixed CRS model that computes a related function $g$ (derived from $f$ ). Protocol $\Sigma$ guarantees security in the $\mathcal{F}_{C R S}$-hybrid model when either party is corrupted, and security in the $\mathcal{F}_{m C R S}$-hybrid model when the second party is corrupted. In the second stage of our proof, we show that one of the parties running $\Sigma$ can run a successful split simulator strategy 8] against the other. As in [8], the existence of a split simulator strategy means that the class of functionalities that can be securely realized by the two-party protocol $\Sigma$ is severely restricted. This also restricts the class of functionalities $f$ which can be realized using the original $n$-party protocol.

We now give the details. Let $x \| y$ denote the concatenation of $x$ and $y$. We first define the $t$-division of a function $f$.

Definition 2. Let $f=\left(f_{1}, \ldots, f_{n}\right)$ be a function taking $n$ inputs $x_{1}, \ldots, x_{n}$ and returning $n$ (possibly different) outputs. Define the two-input/two-output function $g=\left(g_{1}, g_{2}\right)$, the $t$-division of $f$ via:

$$
\begin{aligned}
g_{1}(\overbrace{\left(x_{1}\|\cdots\| x_{t}\right)}^{I_{1}}, \overbrace{\left(x_{t+1}\|\cdots\| x_{n}\right)}^{I_{2}}) & =f_{1}\left(x_{1}, \ldots, x_{n}\right)\|\cdots\| f_{t}\left(x_{1}, \ldots, x_{n}\right) \\
g_{2}\left(\left(x_{1}\|\cdots\| x_{t}\right),\right. & \left.\left(x_{t+1}\|\cdots\| x_{n}\right)\right)=f_{t+1}\left(x_{1}, \ldots, x_{n}\right)\|\cdots\| f_{n}\left(x_{1}, \ldots, x_{n}\right) .
\end{aligned}
$$

Lemma 1. Let $n, t, s$ be such that $s+t=n$ and $s<n / 2$. Say $\Pi$ is an $(s, t)$ secure protocol by which parties $P_{1}, \ldots, P_{n}$ holding inputs $x_{1}, \ldots, x_{n}$ can evaluate a function $f\left(x_{1}, \ldots, x_{n}\right)$. Then there exists a two-party protocol $\Sigma$ by which parties $p_{1}, p_{2}$ holding inputs $I_{1}=x_{1}\|\ldots\| x_{t}$ and $I_{2}=x_{t+1}\|\ldots\| x_{n}$ can evaluate the $t$-division function $g\left(I_{1}, I_{2}\right)$. Furthermore, $\Sigma$ is secure when either parties is corrupted in the $\mathcal{F}_{C R S}$-hybrid model, and secure against a dishonest $p_{2}$ in the $\mathcal{F}_{\text {mCRS }}$-hybrid model.

Proof. We construct the protocol $\Sigma$ using the protocol $\Pi$. The basic idea is as follows. The parties $p_{1}$ and $p_{2}$ break their input $I_{1}, I_{2}$ into several parts and start 
emulating $n$ parties running the protocol $\Pi$ to compute $f$ on those inputs. Some of these parties in $\Pi$ are controlled and emulated by $p_{1}$ and others by $p_{2}$. Finally when $\Pi$ finishes, $p_{1}$ and $p_{2}$ get several outputs $f_{i}$ meant for parties controlled by them. Using these outputs, $p_{1}$ and $p_{2}$ then individually reconstruct their final output $g_{1}$ and $g_{2}$. More details follow.

The parties $p_{1}, p_{2}$ hold inputs $I_{1}=x_{1}\|\ldots\| x_{t}$ and $I_{2}=x_{t+1}\|\ldots\| x_{n}$ and wish to compute the function $g$. Party $p_{1}$ internally starts emulating parties $P_{1}, \ldots, P_{t}$ on inputs $x_{1}, \ldots, x_{t}$, respectively, to compute the function $f$. Similarly, $p_{2}$ starts emulating parties $P_{t+1}, \ldots, P_{n}$ on inputs $x_{t+1}, \ldots, x_{n}$. Whenever $\Pi$ requires party $P_{i}$ to send a message $M$ to party $P_{j}$, this is handled in the natural way: If $i, j \leq t$ (resp., $i, j>t$ ), then $p_{1}$ (resp., $p_{2}$ ) internally delivers $M$ from $P_{i}$ to $P_{j}$. If $i \leq t$ and $j>t$, then $p_{1}$ sends the message $(i, j, M)$ to $p_{2}$ who then internally delivers $M$ to $P_{j}$ as if it were received from $P_{i}$. The case $i>t$ and $j \leq t$ is handled similarly. After $\Pi$ finishes, $P_{1}, \ldots, P_{t}$ halt outputting $f_{1}, \ldots, f_{t}$ and hence $p_{1}$ obtains $g_{1}=f_{1}\|\ldots\| f_{t}$. Similarly, $p_{2}$ obtains $g_{2}=f_{t+1}\|\ldots\| f_{n}$.

As for the security claims regarding $\Sigma$, recall that $\Pi$ is $t$-secure in the $\mathcal{F}_{\mathrm{CRS}}-$ hybrid model. This means that $\Pi$ securely computes $f$ in the presence of any coalition of up to $t$ corrupted parties. This in particular means that $\Pi$ remains secure if all of $P_{1}, \ldots, P_{t}$ are corrupted. Thus, $\Sigma$ remains secure against a dishonest $p_{1}$ (who controls $P_{1}, \ldots, P_{t}$ ) in the $\mathcal{F}_{\mathrm{CRS}}$-hybrid model. Also since $s \leq t$ (because $s<n / 2$ ), protocol $\Pi$ is secure even if $P_{t+1}, \ldots, P_{n}$ are corrupted and hence $\Sigma$ is secure against a dishonest $p_{2}$ in the $\mathcal{F}_{\mathrm{CRS}}$-hybrid model. Furthermore, $\Pi$ is $s$-secure in the $\mathcal{F}_{m C R S}$-hybrid model. This means that $\Pi$ remains secure even if $P_{t+1}, \ldots, P_{n}$ are corrupted. Hence $\Sigma$ is secure against a dishonest $p_{2}$ (but not necessarily against a dishonest $p_{1}$ ) in the $\mathcal{F}_{m C R S}$-hybrid model.

We now show that a malicious $p_{2}$ can run a successful split simulator strategy [8] against an honest $p_{1}$ in protocol $\Sigma$ when run in the $\mathcal{F}_{m C R S}$-hybrid model. This shows that even if $p_{1}$ remains honest, there is a large class of functionalities that cannot be securely realized by $\Sigma 2$ Using the previous lemma, this in turn shows the existence of a class of functionalities which cannot be $(s, t)$-securely realized by $\Pi$ (when $t+s \geq n)$.

Showing the existence of a successful split simulator strategy for $p_{2}$ amounts to reproving the main technical lemma of [8] in our setting. We start by recalling a few definitions and notations from 98. Part of our proof is taken almost verbatim from [8].

Notation. Let $g: D_{1} \times D_{2} \rightarrow\{0,1\}^{*} \times\{0,1\}^{*}$ be a deterministic, polynomialtime computable function, where $D_{1}, D_{2} \subseteq\{0,1\}^{*}$ are arbitrary (possibly infinite) domains of inputs. Function $g$ is denoted by $g=\left(g_{1}, g_{2}\right)$ where $g_{1}$ and $g_{2}$ denote the outputs of $p_{1}$ and $p_{2}$, respectively. The following definition corresponds to [8, Def. 3.1].

${ }^{2}$ In $\left[8\right.$, it was shown that either party $p_{1}$ or $p_{2}$ could run a split simulator strategy against the other. In our case, we only show that $p_{2}$ can do so against $p_{1}$. Hence, the class of functionalities which we prove are impossible to realize is smaller than that in 8 . 
Definition 3. Let $\Sigma$ be a protocol securely computing g. Let $D_{\kappa} \subseteq D_{2}$ be a polynomial-size subset of inputs (i.e., $\left|D_{\kappa}\right|=\operatorname{poly}(\kappa)$, where $\kappa$ is a security parameter). Then a corrupted party $p_{2}$ is said to run a split adversarial strategy if it consists of machines $p_{2}^{a}$ and $p_{2}^{b}$ such that:

1. On input $\left(1^{\kappa}, D_{\kappa}, I_{2}\right)$, with $I_{2} \in D_{\kappa}$, party $p_{2}$ internally gives machine $p_{2}^{b}$ the input $\left(1^{\kappa}, D_{\kappa}, I_{2}\right)$.

2. An execution between (an honest) $p_{1}$ running $\Sigma$ and $p_{2}=\left(p_{2}^{a}, p_{2}^{b}\right)$ works as follows:

(a) $p_{2}^{a}$ interacts with $p_{1}$ according to some specified strategy.

(b) At some stage of the execution $p_{2}^{a}$ hands $p_{2}^{b}$ a value $I_{1}^{\prime}$.

(c) When $p_{2}^{b}$ receives $I_{1}^{\prime}$ from $p_{2}^{a}$, it computes $J_{1}^{\prime}=g_{1}\left(I_{1}^{\prime}, I_{2}^{\prime}\right)$ for some $I_{2}^{\prime} \in D_{\kappa}$ of its choice.

(d) $p_{2}^{b}$ hands $p_{2}^{a}$ the value $J_{1}^{\prime}$, and $p_{2}^{a}$ continues interacting with $p_{1}$.

We define a successful strategy as in [8, Def. 3.2].

Definition 4. Let $\Sigma, g, \kappa$ be as in Definition 3. Let $\mathcal{Z}$ be an environment who hands input $I_{1}$ to $p_{1}$ and a pair $\left(D_{\kappa}, I_{2}\right)$ to $p_{2}$ where $D_{\kappa} \subseteq D_{2},\left|D_{\kappa}\right|=\operatorname{poly}(\kappa)$, and $I_{2}$ is chosen uniformly in $D_{\kappa}$. Then a split adversarial strategy for $p_{2}$ is said to be successful if for every $\mathcal{Z}$ as above and every input $z$ to $\mathcal{Z}$, the following conditions hold in a real execution of $p_{2}$ with $\mathcal{Z}$ and honest $p_{1}$ :

1. The value $I_{1}^{\prime}$ output by $p_{2}^{a}$ in step $2 b$ of Definition 3 is such that for every $I_{2} \in D_{\kappa}$, it holds that $g_{2}\left(I_{1}^{\prime}, I_{2}\right)=g_{2}\left(I_{1}, I_{2}\right)$.

2. The honest party $p_{1}$ outputs $g_{1}\left(I_{1}, I_{2}^{\prime}\right)$, where $I_{2}^{\prime}$ is the value chosen by $p_{2}^{b}$ in step 2c of Definition 3 .

We now prove a lemma akin to [8, Lem. 3.3].

Lemma 2. Let $\Sigma$ be a non-trivial, two-party protocol computing g, which is secure in the $\mathcal{F}_{C R S}$-hybrid model when either party is corrupted, and secure in the $\mathcal{F}_{m C R S}$-hybrid model when $p_{2}$ is corrupted. Then there exists a machine $p_{2}^{a}$ such that for every machine $p_{2}^{b}$ of the form described in Definition 3, the split adversarial strategy $p_{2}=\left(p_{2}^{a}, p_{2}^{b}\right)$ is successful in the $\mathcal{F}_{m C R S}$-hybrid model, except with negligible probability.

Proof. The proof in our setting is very similar to the proof of the main technical lemma in [8]. Here we only sketch a proof, highlighting the main differences. We refer the reader to 8 for complete details.

In the proof of [8], they first consider the real-world execution where party $p_{1}$ is controlled by the environment $\mathcal{Z}$ through a dummy adversary $\mathcal{A}_{D}$ who simply forwards messages received from the environment to party $p_{2}$ and vice versa. Parties $p_{1}$ and $p_{2}$ have inputs $I_{1}$ and $I_{2}$, respectively, and execute $\Sigma$; we assume that $\Sigma$ securely computes $g$. Thus, there exists a simulator $\mathcal{S}$ that interacts with the ideal process and such that $\mathcal{Z}$ cannot distinguish an execution of a real-world process from an execution of the ideal process. Notice that in the ideal world, $\mathcal{S}$ must send an input $I_{1}^{\prime}$ to the ideal functionality computing $g$, and receives an 
output $J_{1}^{\prime}$ from this functionality such that $I_{1}^{\prime}$ and $J_{1}^{\prime}$ are functionally equivalent to $I_{1}$ and $g_{1}\left(I_{1}, I_{2}^{\prime}\right)$ respectively. (Here, $I_{2}^{\prime}$ is chosen by $p_{2}$.) This implies that if $\mathcal{Z}$ simply runs the code of an honest $p_{1}$, the ideal-world simulator $\mathcal{S}$ is able to extract the inputs of the honest player $p_{1}$ and also force its output to be $J_{1}^{\prime}$.

In our setting, in the $\mathcal{F}_{\mathrm{CRS}}$-hybrid model (i.e., if the string $\sigma$ is an honestlygenerated CRS), protocol $\Sigma$ is secure regardless of which party is corrupted. This means that there exists a simulator $\mathcal{S}$ who generates a CRS $\sigma$ and is then able to extract the input of the honest player $p_{1}$.

Now consider the case of the $\mathcal{F}_{m C R S}$-hybrid model, i.e., when $\Sigma$ is run with an adversarially-generated string $\sigma$. In this case, a malicious $p_{2}$ can just run $\mathcal{S}$ to generate a CRS and interact with $p_{1}$. At a high level, the machine $p_{2}^{a}$ just consists of running $\mathcal{S}$ with the honest $p_{1}$. Machine $p_{2}^{a}$ forwards every message that it receives from $p_{1}$ to $\mathcal{S}$ as if it came from $\mathcal{Z}$. Similarly, every message that $\mathcal{S}$ sends to $\mathcal{Z}$ is forwarded by $p_{2}^{a}$ to $p_{1}$ in the real execution. When $\mathcal{S}$ outputs a value $I_{1}^{\prime}$ that it intends to send to the ideal functionality computing $g$, then $p_{2}^{a}$ gives this value to $p_{2}^{b}$. Later, when $p_{2}^{b}$ gives a value $J_{1}^{\prime}$ to $p_{2}^{a}$, then $p_{2}^{a}$ gives it to $\mathcal{S}$ as if it came from the ideal functionality computing $g$. Hence, a malicious $p_{2}$ is able to use the simulator $\mathcal{S}$ to do whatever the simulator $\mathcal{S}$ was doing in the

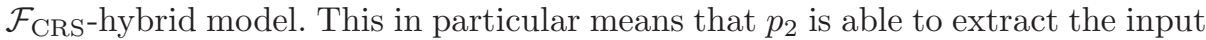
of the honest $p_{1}$ and run a successful split simulator strategy. This completes our proof sketch.

Completing the proof of Theorem 2, As shown by [8, the existence of a successful split simulator strategy for $p_{2}$ against an honest $p_{1}$ rules out the realization of several interesting well-formed functionalities. This, in turn, rules out several $n$-input functionalities $f$ whose secure computation implies secure computation of $g$ by Lemma 1. We give a concrete example in what follows.

We consider single-input functions which are not efficiently invertible [8]. The definition of an efficiently-invertible function is given as in [8]:

Definition 5. A polynomial-time function $g: D \rightarrow\{0,1\}^{*}$ is efficiently invertible if there exists a PPT machine $M$ such that for every distribution $\hat{D}=\left\{\hat{D}_{\kappa}\right\}$ over $D$ that is sampleable by a non-uniform, PPT Turing machine, the following is negligible:

$$
\underset{x \leftarrow \hat{D}_{\kappa}}{\operatorname{Pr}}\left[M\left(1^{\kappa}, g(x)\right) \notin g^{-1}(g(x))\right] .
$$

Let $t, s, n$ be such that $t+s=n$ and $s<n / 2$. We consider the following functionality $\mathcal{F}$ : Let parties $P_{1}, \ldots, P_{t}$ hold inputs $x_{1}, \ldots, x_{t}$, while $P_{t+1}, \ldots, P_{n}$ have no inputs. The output of $P_{1}, \ldots, P_{t}$ is $\perp$ while the output of $P_{t+1}, \ldots, P_{n}$ is $f\left(x_{1}\|\cdots\| x_{t}\right)$ for an function $f$ which is not efficiently invertible.

If there exists an $n$-party protocol $\Pi$ that $(s, t)$-securely realizes $\mathcal{F}$, then there exists a 2-party protocol $\Sigma$ computing the function $g\left(I_{1}, \perp\right)=\left(\perp, f\left(I_{1}\right)\right)$, which is secure against corruption of either party in the $\mathcal{F}_{\mathrm{CRS}}$-hybrid model and secure against corruption of the second party in the $\mathcal{F}_{m C R S}$-hybrid model. Lemma 2, however, implies that $p_{2}$ can run a successful split simulator strategy and extract an input $I_{1}^{\prime}$ such that $g\left(I_{1}, \perp\right)=g\left(I_{1}^{\prime}, \perp\right)$, or equivalently $f\left(I_{1}\right)=f\left(I_{1}^{\prime}\right)$. Since 
all the information computable by $p_{2}$ during an execution of $\Sigma$ should follow from its output $f\left(I_{1}\right)$ alone, it follows that $I_{1}^{\prime}$ is computable given $f\left(I_{1}\right)$. This contradicts the assumption that $f$ is not efficiently invertible.

Hence, we conclude that there does not exist such a protocol $\Pi$ to evaluate the functionality $\mathcal{F}$. This impossibility result can be extended to include a large class of functionalities as in 8 .

\section{References}

1. Barak, B., Canetti, R., Nielsen, J.B., Pass, R.: Universally composable protocols with relaxed set-up assumptions. In: 45th Annual Symposium on Foundations of Computer Science (FOCS), pp. 186-195. IEEE, Los Alamitos (2004)

2. Barak, B., Sahai, A.: How to play almost any mental game over the net - concurrent composition using super-polynomial simulation. In: 46th Annual Symposium on Foundations of Computer Science (FOCS), IEEE, Los Alamitos (2005)

3. Ben-Or, M., Goldwasser, S., Wigderson, A.: Completeness theorems for noncryptographic fault-tolerant distributed computation. In: 20th Annual ACM Symposium on Theory of Computing (STOC), pp. 1-10. ACM, New York (1988)

4. Blum, M., Feldman, P., Micali, S.: Non-interactive zero-knowledge and its applications. In: 20th Annual ACM Symposium on Theory of Computing (STOC), pp. 32-42. ACM, New York (1988)

5. Canetti, R.: Universally composable security: A new paradigm for cryptographic protocols. In: 42nd Annual Symposium on Foundations of Computer Science (FOCS), pp. 136-147. IEEE, Los Alamitos (2001) Preliminary full version available as Cryptology ePrint Archive Report 2000/067

6. Canetti, R., Dodis, Y., Pass, R., Walfish, S.: Universally composable security with global setup. In: Vadhan, S.P. (ed.) TCC 2007. LNCS, vol. 4392, pp. 6185. Springer, Heidelberg (2007)

7. Canetti, R., Fischlin, M.: Universally composable commitments. In: Kilian, J. (ed.) CRYPTO 2001. LNCS, vol. 2139, pp. 19-40. Springer, Heidelberg (2001)

8. Canetti, R., Kushilevitz, E., Lindell, Y.: On the limitations of universally composable two-party computation without set-up assumptions. J. Cryptology 19(2), 135-167 (2006)

9. Canetti, R., Lindell, Y., Ostrovsky, R., Sahai, A.: Universally composable twoparty and multi-party secure computation. In: 34th Annual ACM Symposium on Theory of Computing (STOC), pp. 494-503 (2002)

10. Goldwasser, S., Lindell, Y.: Secure multi-party computation without agreement. J. Cryptology 18(3), 247-287 (2005)

11. Groth, J., Ostrovsky, R.: Cryptography in the multi-string model. In: Menezes, A. (ed.) CRYPTO 2007. LNCS, vol. 4622, pp. 323-341. Springer, Heidelberg (2007)

12. Hofheinz, D., Müller-Quade, J., Unruh, D.: Universally composable zero-knowledge arguments and commitments from signature cards. In: Proc. 5th Central European Conference on Cryptology (2005)

13. Ishai, Y., Kushilevitz, E., Lindell, Y., Petrank, E.: On combining privacy with guaranteed output delivery in secure multiparty computation. In: Dwork, C. (ed.) CRYPTO 2006. LNCS, vol. 4117, pp. 483-500. Springer, Heidelberg (2006) 
14. Katz, J.: On achieving the best of both worlds in secure multiparty computation. In: 39th Annual ACM Symposium on Theory of Computing (STOC), pp. 11-20. ACM, New York (2007)

15. Katz, J.: Universally composable multi-party computation using tamper-proof hardware. In: Naor, M. (ed.) EUROCRYPT 2007. LNCS, vol. 4515, pp. 115-128. Springer, Heidelberg (2007)

16. Prabhakaran, M., Sahai, A.: New notions of security: Achieving universal composability without trusted setup. In: 36th Annual ACM Symposium on Theory of Computing (STOC), pp. 242-251 (2004)

17. Rabin, T., Ben-Or, M.: Verifiable secret sharing and multi-party protocols with honest majority. In: 21st Annual ACM Symposium on Theory of Computing (STOC), pp. 73-85. ACM, New York (1989) 